\title{
Cryptorchidism and its impact on male fertility: a state of art review of current literature
}

\author{
Eric Chung, MBBS, FRACS; Gerald B. Brock, MD, FRCSC
}

Division of Urology, University of Western Ontario, London, ON

Cite as: Can Urol Assoc J 201 1;5(3):210-4; D01:10.5489/cuaj.10106

\section{Abstract}

Cryptorchidism is associated with impairment of germ cell maturation and subsequent infertility in adulthood. The present report details common scenarios of referral of men with prior history of cryptorchidism and orchidopexy seeking advice for infertility, and examines the association between cyrptorchidism and male infertility. The increase in the understanding of the hormonal profiles and patho-physiological changes in germ cell maturation in cryptorchid boys may potentially change our approach and management strategies. Improvement in sperm retrieval techniques and micromanipulation techniques, such as intracytoplasmic sperm injection, has led to excellent fertilization and pregnancy outcomes of treatment cycles.

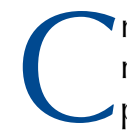

ryptorchidism or undescended testis is one of the most common congenital conditions in the pediatric population. The prevalence of cryptorchidism in full-term newborns ranges between $1 \%$ and $3 \%$, while in premature boys it is reported to be as high as $30 \%{ }^{1}$ Impairment of germ cell maturation and subsequent infertility in adulthood are well-recognized consequences of cryptorchidism. The incidence of azoospermia in unilateral cryptorchidism is $13 \%$ and this figure increases to $89 \%$ in untreated bilateral cryptorchidism, making cryptorchidism the most common etiologic factor of azoospermia in the adult. ${ }^{2}$ Reports have suggested that orchidopexy be performed before 1 year of age to minimize germ cell loss as fertility index (number of spermatogonia per tubule) is adversely affected in boys with cryptorchid testes beyond a year. ${ }^{3,4}$

Over the past decade, the rapid development of novel management approaches for male factor infertility has enabled many of these men, previously believed to be infertile, the ability to become fathers. Infertility or subfertility affects $15 \%$ of couples in Canada, and about $50 \%$ of fertility problems are attributed to a male factor. Of these men, testicular failure or non-obstructive azoospermia is the predominant component. Testicular spermatozoa can be retrieved in some non-obstructive azoospermia men despite the absence of ejaculated spermatozoa in their semen, for instance in cryptozoospermic men (intermittent detection of spermatozoa in ejaculate) due to isolated foci of active spermatogenesis. Further improvement in sperm retrieval techniques has allowed infertile couples to become engaged in the discussion of their potential for fertility.

Micromanipulation techniques, such as intracytoplasmic sperm injection (ICSI) applied to sperm and ova in vitro (in-vitro fertilisation [IVF]), allow sperm with limited intrinsic fertilizing capacity to produce viable embryos. Since the introduction of microsurgical sperm retrieval and ICSI as accepted treatments for the azoospermic man, many studies have reported excellent fertilization and pregnancy outcomes of treatment cycles.

The present report details 2 common scenarios of referral to our tertiary care fertility clinic of men with prior history of cryptorchidism and orchidopexy seeking advice for infertility.

\section{Case 1}

A 33-year-old man, with his healthy 28-year-old partner, had been trying to conceive. He underwent a bilateral orchidopexy at the age of 8 . While the records of intraoperative findings were not available, he recalls no postoperative complications. He reported no prior farm work or exposure to chemical pesticides, denied smoking or recreational drug use, and does not have history of sexually transmitted infections. He voids normally without history of urinary tract infection, epididymitis or other genitourinary trauma and has no erectile or ejaculatory issues. He does not take any medication or had any other surgery apart from bilateral orchidopexy. According to his parents, the operation was 
uneventful and he did not have any postoperative complication. He recalls normal growth and development similar to his male siblings. He has 2 older brothers; both have 2 children each. He works as a computer analyst and travels by car.

He has been in a relationship with his current female partner for 3 years and they have been trying to conceive for 2 years. He has normal libido and sexual desire and is able to achieve satisfactory erection and normal ejaculate. His female partner has been assessed by the gynecologist and no contributing female factors were detected.

Physical examination revealed a fit healthy man with normal secondary sexual characteristics. Testes, epididymis and vas were normal on palpation with no evidence of varicocele. Digital rectal examination was normal. Laboratory tests showed azoospermia with normal volume and $\mathrm{pH}$ on 2 semen-analysis samples. His follicle-stimulating hormone $(\mathrm{FSH})$ was elevated $26 \mathrm{IU} / \mathrm{L}$, while his luteinizing hormone $(\mathrm{LH})$ and testosterone were within normal range. Both genetic karyotyping and $\mathrm{Y}$-chromosome micro-deletion were also normal. Ultrasound $(\mathrm{U} / \mathrm{S})$ of his scrotum was unremarkable.

The young man decided to undergo testicular biopsy for diagnostic purpose instead of sperm retrieval for ICSI preparation and cryopreservation, since the young couple is keen to avoid the high cost associated with the latter procedure. Testicular sperm extraction (TESE) was performed under local anaesthesia and more than $30 \%$ of the testicular tissue was removed. The testicular tissue appeared soft and brownish in characteristic. The tunica albuginea of testis, scrotal dartos and skin were closed in separate layers. The histopathology revealed Sertoli cell only in the testicular tissue.

At a subsequent follow-up visit, the couple was counselled with regards to the operative findings and advised to consider a sperm donor or adoption.

\section{Case 2}

A 29-year-old man was referred by a gynecologist who had been assessing him and his partner for fertility. While his 26-year-old female partner had no fertility issues, this young man was found to have severe oligospermia on the initial semen analysis. Despite strict adherence to abstinence 72 hours prior and taking over-the-counter vitamin supplements, the repeat semen analysis confirmed isolated severe oligospermia.

He reported a history of right orchidopexy when he was 6 months old. During his college years, he had been kicked on the groin area several times during sporting activities, but denied any significant pain, scrotal swelling or traumatic history requiring emergency presentation or surgical intervention. He reported normal growth and development.
He smokes 1 package of cigarettes daily with a 5 pack/year smoking history. As a physically fit man, he did not have any other risk factor for male infertility. At present, he is undertaking a Master's Degree in human physiology. He has been married to his partner for 2 years and they have been trying to conceive for the past 18 months.

Physical examination revealed right inguinal scar with normal testes size. He exhibited normal secondary sexual characteristics and no genital abnormalities were found. Laboratory tests showed 2 million sperm/cc with 30\% motility and $<50 \%$ demonstrating normal morphology. The ejaculate volume and $\mathrm{pH}$ were within normal range. A repeat semen analysis performed 1 month later showed 3.5 million sperm/cc with $30 \%$ motility and $<50 \%$ normal morphology. His serum FSH, LH and testosterone, as well as scrotal U/S, were normal. Further investigations, such as sexual chromosomal abnormalities and $\mathrm{Y}$-chromosome micro-deletion study, were also normal.

Following a discussion between the young couple and the gynaecologist, the decision was taken to undergo sperm retrieval for consideration of ICSI and IVF, rather than a testicular biopsy for histopathology. Blood screening for human immunodeficiency virus I and II, hepatitis B and C, syphilis and human T-lymphotrophic virus I and II were carried out. The young man was counselled with regards to the process of sperm retrieval and the costs of the procedure, sperm cryopreservation and storage. Sperm retrieval was performed under local anesthetic with percutaneous epididymal sperm aspiration (PESA) as the firstline evaluation. Unfortunately, the PESA did not detect any spermatozoa and a TESE was then carried out. Microscopic surgical dissection of the seminiferous tubules revealed the presence of sufficient spermatozoa; the extracted sperm was prepared and cultured for 24 hours to induce sufficient sperm motility. Then the motile sperm was stored frozen in several micropipettes overnight and a sample of the frozen micropipette was thawed the following day to ensure that the sperm remained viable and motile. The confirmation of viable and motile sperm led his partner to undergo an IVF treatment cycle.

\section{Discussion}

Cryptorchidism is a common developmental abnormality. Testicular development requires the presence of the sex (testis) determining region on $Y$ chromosome (SRY gene) for normal differentiation. Testicular tissue arises from differentiation of the gonadal ridge and an intact hypothalamic-pituitary-gonadal axis is a prerequisite for the testicular descent. ${ }^{5}$ Although the incidence of cryptochidism in full-term boys ranges between $1 \%$ and $3 \%$, the prevalence decreases to $0.8 \%$ and $1.5 \%$ at 1 year of age. ${ }^{6}$ Several factors are associated with cryptorchidism, such as prematurity, low birth 
weight, familial and maternal exposure to estrogen, during the first trimester.

About $80 \%$ of undescended testes are palpable and $20 \%$ are non-palpable. ${ }^{5}$ It is important to differentiate true cryptochidism from other conditions, such as retractile testis, absent or vanishing testis and ectopic testis. Most palpable undescended testes are located along the inguino-scrotal region with most intra-abdominal testes found within a few centimetres of the internal ring. In general, ductal abnormalities and testicular maldevelopment are more common in boys with abdominal testes. The incidence of unilateral undescended testes is more common than bilateral undescended testes. The main reasons for the treatment of undescended testes are increase fertility and decrease the risk of testicular torsion or injury and testicular cancer, as well as psychological stigma.

Cryptochidism should be treated when the patient is about 6 months old due to the rarity of spontaneous descent after this time and the possible improvements in fertility that early intervention may confer. ${ }^{1,7}$ The choice of initial treatment is a reflection of the preferences of treating team, the patient and his family. Hormonal therapies, such as human chorionic gonadotropin (HCG), gonadotropin (LH)releasing hormone $(\mathrm{GnRH}, \mathrm{LHRH})$ or in combination, have an overall success rate of less than $25 \%$; the success depends on the pre-treatment location of testis. ${ }^{7,8}$ On the other hand, surgical orchidopexy is associated with a success rate higher than $80 \%{ }^{9}$

The relationship between cryptorchidism and male infertility is evident when azoospermic men were studied. About $10 \%$ of infertile men have a history of cryptorchidism and orchidopexy. ${ }^{10}$ Bilateral cryptorchid men have a 6 -fold greater risk of being infertile when compared with unilaterally cryptorchid men and general population. ${ }^{11}$ The incidence of azoospermia in men with unilateral cryptorchidism is $13 \%$ regardless of the fate of the testis. Among men with untreated bilateral cryptorchidism, 98\% develop azoospermia. Interestingly, the risk of azoospermia decreases to $32 \%$ among patients treated medically and $46 \%$ in men who underwent orchidopexy as a child. ${ }^{2}$ Therefore, the question arises on whether there is inherent irreversible damage in spermatogenesis. If so, how can we assess the fertility of cryptorchid males to better counsel them with respect to future fertility?

The reduction in germ cell count starts as early as 6 months of age and is dependent on the position of the testis. In general, the higher the testicular position at the time of treatment, the fewer the number of germ cells. ${ }^{12}$ Testicular biopsy at the time of orchidopexy can provide significant information on the cryptorchid men future fertility. Spermatogenic index decreases significantly by 9 months of age and orchidopexy at or before this time may stop testicular degeneration in selected individuals and may improve their chance of preserving fertility.

Non-palpable testes are associated with greater germ cell loss than clinically palpable testes. In cryptorchidism, Leydig cell atrophy is prominent in life due to insufficient gonadotropic secretion. Tasian and colleagues reported a significant $2 \%$ risk per month of severe germ cell loss and $1 \%$ risk per month Leydig cell depletion for each month a testis remains undescended; they also reported a $50 \%$ greater risk of germ cell depletion in non-palpable relative to palpable cryptorchid testes. ${ }^{13}$ The odds of germ cell loss almost double for each age range at the time of orchidopexy. Interestingly, the study did not find any correlation between the presence of unilateral or bilateral disease on the pathological outcomes.

Previously it was thought that the contralateral descended testis in a cryptorchid male should be normal and, therefore, should provide a fertility rate close to the normal male population. Also, if the man underwent orchidopexy at an early age and the testicular biopsy demonstrated the presence of normal germ cells, the patient should technically be fertile. However, early orchidopexy does not always guarantee later fertility. Hadziselimovic and colleagues have shown that despite orchidopexy before 6 months, up to $35 \%$ of boys will grow up to be infertile, even though they had normal number of germ cells at the time of surgery. ${ }^{14}$ The authors postulated that the infertility is caused by a defective transformation of germ cells into Ad (dark) spermatogonia, the progenitor of spermatozoa. During the first 3 to 6 months of age, there is a temporary surge in gonadotropins ( $\mathrm{FSH}$, $\mathrm{LH}$ and inhibin B) and testosterone (i.e., mini-puberty) and results in transforming germ cells (gonocytes) to Ad spermatogonia. ${ }^{7,14,15}$ Hadziselimovic and colleagues also showed that the number of Ad spermatogonia in unilateral cryptochid testes correlated in a nonlinear fashion with those in the contralateral testes. ${ }^{14} \mathrm{Also}$, if this transformation does not occur during this mini-puberty period, the risk of later infertility approaches $90 \%{ }^{2}$ This defect in the first stage of germ cell maturation leads to a disproportionate decrease in Ad spermatogonia and may explain the high rate of infertility in cryptorchid patients with normal number of germ cells at the time of surgery. Hadziselimovic contends that cryptoorchid boys who are non-responders to LHRH therapy should receive a testicular biopsy at the time of orchidopexy to document the presence of germ cell in seminiferous tubules and the total numbers of Ad spermatogonia. ${ }^{7}$ This strategy ensures the identification of those who would benefit from LHRH treatment following orchidopexy.

Studies of hormonal profiles have documented different levels of inhibin B, FSH and LH levels with the level of sperm density and spermatogenesis. The FSH levels in men are usually inversely correlated with sperm counts and indicate the integrity of seminiferous tubules, a marker of spermatogenesis. ${ }^{16}$ Patients with bilateral undescended testes 
have significantly lower sperm motility, sperm concentration, testicular volume and inhibin B levels, while showing higher $\mathrm{FSH}$ and $\mathrm{LH}$ levels than those with unilateral undescended testis. ${ }^{16,17}$ Rusnack and colleagues demonstrated that males with unilateral undescended testes were more likely to have higher sperm density and normal hormone profile, while those with bilateral undescended testes had severe oligospermia and abnormal hormonal parameters. ${ }^{18}$ Cortes and colleagues reported while high FSH values were to be expected, normal FSH values were also common in these suspected infertile men. ${ }^{15}$ In men with bilateral undescended testes, a normal FSH is associated with spermatogenic arrest and fewer germ cells, while those with elevated FSH (possible hypergonadotrophic hypogonadism) often have Sertoli cell only syndrome with absent germ cells; men with unilateral undescended testes generally lacked germ cells on testicular biopsies.

Currently, a range of standardized surgical techniques can be used to extract spermatozoa from azoospermic men from their epididymis and/or testis for ICSI. Epididymal sperm retrieval techniques include PESA and microsurgical epididymal sperm aspiration (MESA). Three testicular sperm retrieval techniques have been described: testicular sperm aspiration (TESA), TESE and microdissection-TESE (MD-TESE). Percutaneous sperm aspiration techniques are widely employed owing to their technical facility and relatively low cost. In non-obstructive azoospermia, the finding of testicular spermatozoa constitutes a key element predicting treatment success as only half of these men will have sperm. ${ }^{19}$ Epididymal sperm extraction is not usually feasible in non-obstructive azoospermic men as sperm are rarely present in epididymal tissue.

While there are many factors that could potentially predict successful testicular sperm recovery in the non-obstructive azoospermic man, only testicular histopathology was identified as useful. ${ }^{20,21} \mathrm{~A}$ recent guideline from the Canadian Urological Association advocates that testicular biopsy be offered to azoospermic males in whom the diagnosis will alter management. ${ }^{21}$ The ideal surgical technique for testicular sperm extract should pose minimal trauma to the testis with retrieval of a sufficient amount of motile spermatozoa to inject all available oocytes. The remainder of the retrieved sample can be cryopreserved for further attempts. The TESE with multiple biopsies is considered the optimal option to improve the sperm retrieval rate [SSR] given that isolated regions of spermatogenic tissue are present. ${ }^{22}$ However, the removal of large sample of testicular tissue can lead in some cases to testicular atrophy and potentially impair subsequent success SSR. ${ }^{23}$ Therefore it is advisable that TESE with multiple biopsies should be considered a therapeutic rather than just diagnostic test. In a systematic review of sperm retrieval technique for non-obstructive azoospermia, Donoso and colleagues found TESE with multiple biopsy results in a higher SSR than fine needle aspiration and MD-TESE performs better than conventional TESE only in cases of Sertoli cell only syndrome, where tubules containing active focus of spermatogenesis can be identified. ${ }^{24} \mathrm{MD}$-TESE appears to be the safest technique and reduces the amount of tissue required to retrieve sperm, this technique is not free of complications such as testicular fibrosis and hypogonadism. At present no definitive conclusions can be drawn on the best sperm sperm retrieval technique for non-obstructive azoospermic men based on current cumulative evidence.

\section{Conclusion}

The complete lack of germ cells at the time of surgery is an important predictor of future fertility in the boy with cryptorchidism. The transformation of germ cells into Ad spermatogonia is crucial. Consequently, efforts should be made to preserve germ cell development apart from early orchidopexy. Testicular biopsy should be performed at the time of orchidopexy to identify those who would benefit from LHRH treatment after the procedure. The LHRH analogs induce replication and differentiation of germ cells that enhance the chance of fertility. As patients with bilateral orchidopexy have appreciably worse fertility rate than patients with unilateral orchidopexy, freezing of sperm or testicular tissue with preserved spermatogenesis may need to be considered.

Competing interests: None declared.

This paper has been peer-reviewed.

\section{References}

1. Kolon FT, Patel PR, Huff SD. Cryptorchidism: diagnosis, treatment and long-term prognosis. Urol Clin North Am 2004;31:469-80

2. Hadziselimovic $F$, Herzog B. The importance of both and early orchidopexy and germ cell maturation for fertility. Lancet 2001;358:1156-7.

3. Cortes D, Thorup JM, Visfeldt J. Cryptorchidism: aspect of fertility and neoplasms. A study including data of 1335 consecutive boys who underwent testicular biopsy simultaneously with surgery for cryptorchidim. Horm Res 2001;55:21-5.

4. Trussel JC, Lee PA. The relationship of cryptorchidism to fertility. Curr Urol Rep 2004;5:142-5.

5. Spencer JR. The endocrinology of testicular descent. AUA Update Series Lesson 1994;12:94-9.

6. Leissner J, Filipas D, Wolf HK. The undescended testes: consideration and impact on fertility. BJU Int 1999;83:885-92.

7. Hadziselimovic F. Cryptochidism, its impact on male fertility. Eur Urol 2002;41:121-3.

8. Rajer J, Handelsman DJ, Swerdloff RS, et al. Hormonal therapy of cryptorchidism. A randomised, doubleblind study comparing human chorionic gonadotropin and gonadotropin-releasing hormone. N Eng J Med 1986;314:466-70

9. Docimo SG. The results of surgical therapy for cryptorchidism: a literature review and analysis. J Urol 1995; 154:1148-52.

10. Grasso M, Buonaguidi A, Lania C. Postpubertal cryptorchidism: review and evaluation of fertility. Eur Urol 1991;20:126-8.

11. Lee P. Fertility in cryptorchidism: does treatment make a difference? Endocrinol Metabol Clin North Am 1993:22:479-90. 
Chung and Brock

12. Wilkerson ML, Barton FF, Fox L, et al. Fertility potential: a comparison of intra-abdominal and intracanalicular testes by age groups in children. Horm Res 2001;136:274-6.

13. Tasian GE, Hittleman AB, Kim GE, et al. Age at orchidopexy and testis palpability predict germ cell and leydig cell loss: clinical predictors of adverse histological features of cryptorchidism. J Urol 2009;182:704-9.

14. Hadziselimovic F, Zivkovic D, Bica DTG, et al. The importance of mini-puberty for fertility in cryptochidism. J Urol 2005;174:1536-9.

15. Cortes D, Thorup JM, Lindenberg S, et al. Infertility despite surgery for cryptorchidism in childhood can be classified by patients with normal or elevated follicle stimulating hormone and identified at orchidopexy. BJU Int 2003;91:670-4.

16. Trsinar B, Muravec UR. Fertiligy after unilateral and bilateral orchidopexy for cryptorchidism. World I Urol 2009;27:513-9.

17. Lee PA, Coughlin MT. Fertiligy after bilateral cryporchidism: evaluation of paternity hormone and semen data. Horm Res 2001;55:28-32.

18. Rusnack SL, Wu HY, Huff DS, et al. Testis histopathology in boys with cryptorchidism correlates with future fertility potential. J Urol 2003;169:659-62.
19. Gil-Salom M, Romero J, Minguez Y, et al. Testicular sperm extraction and intracytoplasmic sperm injection: A chance of fertility in non-obstructiive azoospermia. J Urol 1998;160:2063-7.

20. Se JT, Ko WJ. Predictive factors of successful testicular sperm recovery in non-obstuctive azoospermia patients. Int J Androl 2001;24:306-10.

21. Jarvi K, Lo K, Fischer A, et al. CUA Guideline: The workup of azoospermic males. Can Urol Assoc J 2010:4:163-73

22. Hauser $R$, Botchan $A$, Amir $A$, et al. Multiple testicular sampling in non-obstructive azoospermia- is it necessary? Hum Reprod 1998;13:3081-5.

23. Schlegel PN, Su LM. Physiological consequences of testicular sperm extraction. Hum Reprod 1997;12:168892.

24. Donoso P, Tournaye H, Devryoey $P$. Which is the best sperm retrieval technique for non-obstructive azoospermia? A systematic review. Hum Reprod Update 2007;13:539-49.

Correspondence: Dr. Eric Chung, Division of Urology, St. Joseph's Health Care and University of Western Ontario, 268 Grosvenor St., London, 0N N6A 4V2; ericchg@hotmail.com 